\title{
REFERENCES
}

1. N. Aronszajn, Rayleigh-Ritz and A. Weinstein methods for approximation of eigenvalues, Proc. Nat. Acad. Sci. U.S.A. vol. 34 (1948) pp. 474-480 and 594-601.

2. A. Weinstein, New methods for the approximation of eigenvalues, Lectures at the University of Maryland, 1950-1951 (in preparation).

UNIVERSITY OF MARYLAND AND

UNIVERSITY OF ISTANBUL

\section{ON SOME FUNCTIONS HOLOMORPHIC IN AN INFINITE REGION}

\section{YU CHIA-YUNG ${ }^{1}$}

S. Mandelbrojt indicated the following proposition: If a function is holomorphic and bounded in a half-strip of the $z$-plane containing the half-axis $o x$ as a part of its central line and if this function and a certain infinite sequence of its derivatives vanish at the origin, then it is identically zero. The proof of this proposition is based upon a result of Mandelbrojt [1, p. 372]. ${ }^{2}$ In the present paper, we consider a function $F(z)$ holomorphic in a region $\Delta$ of the $z$-plane defined by $x \geqq d,|y| \leqq g(x)$, where $-\infty<d<0$ and where $g(x)$ is a certain positive continuous function tending to zero with $1 / x$. In this case if, in $\Delta, F(z)$ tends to zero rapidly enough and uniformly with respect to $y$ as $x$ tends to infinity, and if $F(z)$ and a certain infinite sequence of its derivatives vanish at the origin, then $F(z)$ is identically zero. In order to establish our proposition, we prove at first a lemma by means of the following theorem of $G$. Valiron $[3$, p. $62, \S 32]$ :

TheORem V. Let $Y(X)$ be a real function having a first derivative for $X \geqq X_{0}$ such that

$$
\lim _{x=\infty} \frac{X Y^{\prime}(X)}{\psi(X)}=1 ; \quad \psi(X) \geqq 1, \quad X \geqq X_{0} ; \quad \lim _{x=\infty} \frac{X \psi^{\prime}(X)}{[\psi(X)]^{2}}=0 .
$$

Let $\Phi(X)$ be an entire function and let $M(r)=\max _{|z|-r}|\Phi(z)|$. Then $a$ necessary and sufficient condition that

Presented to the Society, September 7, 1951; received by the editors May 1, 1951.

${ }^{1}$ The author wishes to express to Professors S. Mandelbrojt and G. Valiron his respectful gratitude for their kind and precious suggestions and criticisms.

2 Numbers in brackets refer to the bibliography at the end of this paper. 


$$
\log M(r) \sim e^{Y(X)}, \quad X=\log r
$$

is that

$$
\nu(r) \sim Y^{\prime}(X) e^{Y(X)} \sim \frac{d}{d X} \log M(r)
$$

where $\nu(r)$ is the rank of the maximum term of the highest rank of the Taylor expansion of $\Phi(z)$ corresponding to the value $|z|=r$.

Lemma. Let $\Phi(z)=\sum_{0}^{\infty} \phi(n) z^{n}$ and let $\mu(r)$ be the value of the maximum terms of $|\phi(n)| r^{n}(n=0,1,2, \cdots)$. If ${ }^{3}$

$$
\mu(r) \sim K\left[\left(\log _{2} r\right)\left(\log _{3} r\right) \cdots\left(\log _{p+1} r\right)\right]^{\log r} \quad(K=\text { const. }>0),
$$

then for any given $\epsilon>0(\epsilon<1)$, we have, for $n$ sufficiently large,

$$
|\phi(n)|<\exp \left\{-\exp \left[\omega_{p}\left(e^{(1-\epsilon) n)}\right]\right\}\right.
$$

and, for a sequence $\left\{n_{k}\right\}$ such that $n_{k+1} / n_{k}$ tends to 1 as $k$ tends to infinity,

$$
\left|\phi\left(n_{k}\right)\right|>\exp \left\{-n_{k} \exp \left[\omega_{p}\left(e^{(1+\epsilon) n_{k}}\right)\right]\right\}
$$

where $p$ is a positive integer and where $\xi=\omega_{p}(\eta)$ is the inverse function of $\eta=\xi(\log \xi)\left(\log _{2} \xi\right) \cdots\left(\log _{p-1} \xi\right)$.

Proof. Since [3, p. 111 and 4, p. 32, chap. II]

$\log M(r) \sim \log \mu(r) \sim(\log r)\left(\log _{3} r+\log _{4} r+\cdots+\log _{p+2} r\right)$,

we have, by Theorem $\mathrm{V}$,

$$
\nu(r) \sim \log \left[\left(\log _{2} r\right)\left(\log _{3} r\right) \cdots\left(\log _{p+1} r\right)\right] .
$$

Considering with Valiron a polygon of Newton and using his notations, we see that

$$
n \sim \log \left[\left(\log _{2} R_{n}\right)\left(\log _{3} R_{n}\right) \cdots\left(\log _{p+1} R_{n}\right)\right] .
$$

$\omega_{p}(\eta)$ being an increasing function, it follows that

$$
\begin{aligned}
\exp \left\{\exp \left[\omega_{p}\left(e^{(1-\epsilon) n}\right)\right]\right\} & <e^{G_{n}}=e^{G_{0}} R_{1} R_{2} \cdots R_{n} \\
& <\exp \left\{n \exp \left[\omega_{p}\left(e^{(1+\epsilon) n}\right)\right]\right\}
\end{aligned}
$$

for $n$ sufficiently large. The lemma will then be completely established by Valiron's reasonings.

The following result is an immediate corollary of our lemma:

${ }^{3}$ We write $\log _{0} x=x$ and $\log _{k}(x)=\log \left(\log _{k-1} x\right), k$ being a positive integer and $x$ being sufficiently large. 
CoROLlaRY. If for a given $\epsilon>0$,

$$
\phi(n)=\exp \left\{-n \exp \left[\omega_{p}\left(e^{(1+\epsilon) n}\right)\right]\right\}
$$

for $n$ sufficiently large, then we have

$$
\mu(r) \leqq\left[\left(\log _{2} r\right)\left(\log _{3} r\right) \cdots\left(\log _{p+1} r\right)\right]^{\log r}
$$

for $r$ sufficiently large.

Now we can prove our main theorem:

THEOREM. Let $g(x)$ be a positive continuous function defined for $x \geqq d(-\infty<d<0)$ decreasing to zero with $1 / x$ for $x$ sufficiently large and satisfying

$$
g(x)=O[g(x+\eta)] \quad(x \rightarrow \infty)
$$

for $|\eta|$ sufficiently small. Denote by $\Delta$ the region of the z-plane defined by $x \geqq d,|y| \leqq g(x)$.

Let $\left\{\nu_{n}\right\}$ and $\left\{q_{n}\right\}$ be two complementary sequences of non-negative integers [1] such that the upper density function [1] $D^{\circ}(q)$ of $\left\{q_{n}\right\}$ satisfies, for $q$ sufficiently large,

$$
D^{*}(q)<\frac{b}{(\log q)\left(\log _{2} q\right) \cdots\left(\log _{p+1} q\right)} \quad\left(0<b=\text { const. }<\frac{1}{2}\right) .
$$

Suppose that $F(z)$ is a function holomorphic in $\Delta$ and satisfying

$$
F^{\left(v_{n}\right)}(0)=0
$$

and, for a given $\epsilon>0$,

$$
F(z)=O\left\{[g(x)]^{\left.\exp \omega_{p}\{g(x)]^{-1-\epsilon}\right\}}\right\} \quad(z \text { in } \Delta, x \rightarrow \infty) .
$$

Then we conclude $F(z) \equiv 0$.

Proof. We can evaluate the moduli of all the derivatives of $F(z)$ on the half-axis $o x: x \geqq 0, y=0$. Let us put

$$
h(x)=\min [x-d, g(\xi)] \quad[x \geqq 0,|x-\xi| \leqq g(x)]
$$

and construct in the $z$-plane circles $C(x):|z-x| \leqq h(x)$ which are evidently situated in $\Delta$. We have

$$
F^{(n)}(x)=\frac{n !}{2 \pi i} \int_{C_{(x)}} \frac{F(z)}{(z-x)^{n+1}} d z \quad(x \geqq 0) .
$$

By hypotheses there exist positive constants $A, B, E$ and $x_{0}>d$ such that: 
$|F(z)| \leqq A$ for $z \in \Delta \cap\left\{\Re(z) \leqq x_{0}+g\left(x_{0}\right)\right\}$;

$|F(z)| \leqq B[g(x)]^{\exp \omega_{p}\left\{[(x)]^{-1-\epsilon}\right\}}$ for $z \in \Delta \cap\left\{\Re(z) \geqq x_{0}-g\left(x_{0}\right)\right\}$;

$g(x)$ decreases for $x \geqq x_{0}-g\left(x_{0}\right)$;

$h(x) \geqq E$ for $x \leqq x_{0}+g\left(x_{0}\right)$.

It follows that

$$
\begin{aligned}
\left|F^{(n)}(x)\right| & \leqq A \cdot \frac{n !}{E^{n}} \text { for } 0 \leqq x \leqq x_{0} ; \\
\left|F^{(n)}(x)\right| & \leqq B n ! \frac{[g(x-g(x))]^{\exp \omega_{p}\left\{[g(x-g(x))]^{-1-\epsilon}\right\}}}{[h(x)]^{n}} \\
& \leqq B n ! \frac{[g(x-g(x))] \exp \omega_{p}\left[(g(x-g(x))]^{-1-\epsilon}\right\}}{[g(x+g(x))]^{n}} \\
& =B n ! \Omega_{n}(x, g(x)), \text { say, for } x \geqq x_{0} .
\end{aligned}
$$

We are going to find an upper bound of $\Omega_{n}(x, g(x))$ for $x \geqq x_{0}-g\left(x_{0}\right)$. By (1),

$$
\Omega_{n}(x, g(x)) \leqq K_{1}^{n} \frac{[g(x)]^{\exp \omega_{p}\left\{[g(x)]^{-1-\epsilon}\right\}}}{[g(x)]^{n}} \quad\left(K_{1}=\text { const. }>0\right) .
$$

For the sake of simplicity, consider the case $g(x)=e^{-x}$. We have

$$
\Omega_{n}(x, g(x)) \leqq K_{1}^{n}\left(e^{-x \exp \omega_{p}(e(1+\epsilon) x)}\right) e^{n x}
$$

for $x \geqq x_{0}-e^{-x_{0}}$. The preceding corollary shows that

$$
\Omega_{n}(x, g(x)) \leqq\left[K_{1}(\log n)\left(\log _{2} n\right) \cdots\left(\log _{p} n\right)\right]^{n}
$$

for integral $x \geqq x_{0}-e^{-x_{0}}$. But, for $0<\delta<1$,

$$
\frac{\exp \left\{-(x+\delta)^{\prime}\left[\exp \omega_{p}\left(e^{(1+\epsilon)(x+\delta)}\right)\right]\right\} e^{n(x+\delta)}}{\exp \left\{-x\left[\exp \omega_{p}\left(e^{(1+\epsilon) x}\right)\right]\right\} e^{n x}} \leqq e^{n} \quad(x \geqq 0) .
$$

Hence we obtain

$$
\Omega_{n}(x, g(x)) \leqq\left[K_{2}(\log n)\left(\log _{2} n\right) \cdots\left(\log _{p} n\right)\right]^{n} \quad\left(K_{2}=\text { const. }>0\right)
$$

for $x \geqq x_{0}-e^{-x_{0}}$ and for $n$ sufficiently large. (We pass from the case $g(x)=e^{-x}$ to the general case simply by replacing $e^{-x}$ in what precedes by $g(x)$.) Consequently we have

$$
\left|F^{(n)}(x)\right| \leqq\left[K_{3}(\log n)\left(\log _{2} n\right) \cdots\left(\log _{p} n\right)\right]^{n} \quad\left(K_{3}=\text { const. }>0\right)
$$

for $x \geqq 0$ and for $n$ sufficiently large. $F(x)$ and its derivatives of lower 
orders are evidently also bounded for $x \geqq 0$. An application of a Mandelbrojt's result on generalized quasi-analy ticity [2, chap. III $]^{4}$ will complete immediately the proof of our theorem.

From this theorem it follows that if $F_{1}(z)$ and $F_{2}(z)$ are functions holomorphic in $\Delta$ and verifying conditions similar to (4) and if $F_{1}^{\left(\nu_{n}\right)}(0)=F_{2}^{\left(\nu_{n}\right)}(0)$ for a sequence $\left\{\nu_{n}\right\}$ defined in the above theorem, then we have $F_{1}(z) \equiv F_{2}(z)$.

We remark that in the case $p=1,(4)$ reduces to

$$
F(z)=O\left\{[g(x)]^{\exp \left\{[g(x)]^{-1-\epsilon}\right\}} \quad(z \text { in } \Delta ; x \rightarrow \infty) .\right.
$$

\section{BIBLIOGRAPHY}

1. S. Mandelbrojt, Sur une inegalité fondamentale, Ann. Ecole Norm. (3) vol. 63 (1946) pp. 351-378.

2. - Séries adherentes, régularisations des suites, applications, Paris, Gauthier-Villars, 1952.

3. G. Valiron, Sur les fonctions entières d'ordre fini et d'ordre nul, Thèse, Paris, 1914, and Annales de la Faculté des Sciences de l'Université de Toulouse vol. 27 (1913) pp. 117-257.

4. - Lectures on the general theory of integral functions, Toulouse, Edouard Prival, 1923; new ed., New York, Chelsea, 1949.

\section{Institut Henri Poincaré}

4 For the case $p=1$ of the mentioned result, see $[1$, p. 372]. 